\title{
Negotiation between the Public and Private: an Observation from Chinese Citizens' Social Conformity and Differentiations during the COVID-19 Pandemic
}

\author{
Xinyan Tao ${ }^{*}$ \\ School of Dulwich International High School, Suzhou, Jiangsu, 215000, China \\ *Corresponding author e-mail: 63838935@qq.com
}

Keywords: social conformity; wearing masks; smoking

\begin{abstract}
With individuals' strengthening awareness of respiratory hygiene during the COVID-19 pandemic, unconventional moral judgment and supervisory mechanisms regarding using masks have aroused controversy on a global scale. The infection control works in the way of efficiency while, on the other hand, citizen supervision lays its disputable moral judgment upon the vague boundary between public and private. If a new citizen's supervision to compel people to follow good respiratory hygiene is established, it can change the basic civil understanding of what is public behavior and what is private behavior. This study aims to discover how this change takes place and its underlying mechanism. Using an online questionnaire that contains both general public judgments and specific private scenario-based questions, the public engagement of Chinese citizens on social supervision of respiratory hygiene is depicted. It is argued according to survey results that civic toleration of not wearing masks are significantly higher when the situations are within private boundaries, suggesting that supervisory mechanism on using masks in case of COVID-19 may successfully become some kind of social conformity yet with quite confusing collective imagination on the private space of citizenship.
\end{abstract}

\section{Introduction}

THE WHO raised its alert level for the COVID-19 in terms of its transmission and influences to 'Very High' at a global level on February 28, 2020. Followed by frequent negative exposures of the coronavirus situation, chaos such as the blind consumption of disinfectant or mask was stimulated, together with pessimistic views upon our socioeconomic ecology reported, which makes the foretell of upcoming social panic not so groundless. Analysis tracing social attribution helps and it could be explained via the Social Amplification of Risk Framework [2], which mentions the effects of secondary factors such as interpretation and credibility of the information on both individuals and the society. Despite the public response, a series of correspond actions then taken by governments, including curfew, quarantine and travel restrictions, have also played a part in the enhanced social panic. As suggested by Stanley Cohen in Folk Devils and Moral Panics, an aspect of social panic refers to 'concern', which is defined as potential threats [3]. The occurrence of social panic is therefore associated with social insecurity, which implies that as regulation on emergency set by governments and public 'concerns' continues, the boundary between our public sphere and the private one might be modified within a relatively short period to some extent.

The technical guidelines on the selection and use of masks issued by the Chinese State Council on February 5, 2020, did give rise to the spread of panic across different populations, shown by the Mask Mania phenomenon. With the government intervention and smooth execution of regulations, the pandemic was soon controlled effectively in China. As reported by the National Health Commission of the People's Republic of China, the number of new confirmed cases of COVID-19 was down to less than a hundred on March 6, 2020, and the number of local borderline cases has reached 0 for the first time on March 18, 2020 [4]. On the other hand, during the same period, other countries such as the United States have reported 10,439 new cases, first time over ten thousand, on March 23, 2020 [5]. The positive media report, no matter based on truth or propaganda, works as a 
cure to public panic. It is worth noting that among all those significant differences in control over the virus across countries, and under certain circumstances, the individuals' attitude towards governmental regulation and other public opinions like mask-wearing and civil supervision resonates with the progress of epidemic [6]. The health emergency needs its cure, while the public understanding of COVID-19 needs another. Sometimes a cure to unite public understandings as one would bring efficiency to the former cure. To our knowledge, no study to date has yielded great success in understanding variations on promotion and stimulation of countrywide respiratory hygiene among different societies through the public attitude way. Thus this should be the focus when this study looks into the details of COVID-19 governmental regulation such as mask-wearing [7].

As has been reported, the administrative intervention in China has shown overall high efficiency in terms of controlling the situation, reducing the number of daily new cases by 3,551 and that of total confirmed cases by 4,234 from February 5, 2020, to March 5, 2020, one month after the introduction of new state guidelines [8,9]. This demonstrates that there has been group pressure exerted on individuals towards mask-wearing. By targeting different mask-wearing situations, this study hopes to investigate the underlying mechanism of social conformity and possible reflection of the public and private sphere accompanied by the formation of group imagination [10].

\section{Methods}

\subsection{Subjects and data collection}

This study's background information was collected based on COVID-19 prevention measures released by WHO, China, and other countries in the world. These versions of prevention guidelines grant mask-wearing different priorities. While people in China started to wear masks in public immediately after the announcement and there soon seemed to be a subconscious value judgment and peer pressure exerted on individuals who chose not to follow the guidelines, other countries initially did not make mask-wearing a priority. The nuances of guidelines then serve as an important basis for the design of the questionnaire, which focuses on civil respiratory hygiene, including mask-wearing and smoking behaviors. In early January 31, 2020 (Beijing time), WHO officially announced 2019-nCoV to be considered Public Health Emergency of International Concern (PHEIC). Nearly at the same time, the National Health Commission of the People's Republic of China issued the guidelines for the use of pneumonia masks to prevent 2019-nCoV. Approximately one month after the announcement, the pandemic in China was overall taken under control, shown by the data from WHO. On the contrary, even other countries had published relevant guidelines respectively regarding respiratory hygiene, with Austria being the first on March 30, 2020, daily confirmed cases were still growing. We, therefore, were concerned about this comparison and hoped to study further into the Chinese population. Through analyzing the release of prevention guidelines in various countries, in particular, the adjustments of the threat level of the virus, we decided to conduct the questionnaire from April 27, 2020, to May 8, 2020. During this period, the COVID-19 threat level in China has not yet been reduced and meanwhile, the global outbreak was worsening, leading multiple countries towards a catastrophic situation. As a result of citizens in all countries paying more attention to the virus, the international information interaction of COVID-19 became relatively frequent. That is to say, to measure Chinese citizens' opinion upon preventions would tell whether there is a unique pattern of public conformity in the global awareness of public health threat.

Chinese citizens of all ages and all occupations who have access to the internet from April 27, 2020, to May 8, 2020, were the target population of the study. They were being told that the questionnaire was investigating public behaviors during the epidemic. Samples were recruited by voluntary sampling via an anonymous online questionnaire of 24 well-designed questions. This could ensure that participants' answers are generally genuine and less likely to be affected by social desirability. The questionnaire was designed into an online form to avoid possible physical distance issues during COVID-19 and cover a wider range of populations. A total of 758 responses were 
received and 743 of them (98.0\%) were then being statistically analyzed. The participants spent approximately an average of 5 minutes 46 seconds, responding the questions and have all provided informed consent to be part of the study.

\subsection{Measures and design}

The questionnaire aims to explore the establishment of civil supervision mechanisms and psychological changes in social conformity in special situations. After gaining the informed consent, the first nine questions were designed to obtain background information of the respondents so that they could be classified according to characteristics such as gender, age and habits. Of all these questions, the third one asked for the respondents' smoking habits. According to the 2019 Chinese Adult Tobacco Survey, $27.7 \%$ of the population reported a habit of smoking, with the smoking rate of males up to $52.1 \%$. Thus we presumed that this characteristic might affect respondents' attitudes towards scenario-based questions and hoped to investigate this variation via subject classification.

While specific situations get embedded with previous judgments made on mask-wearing, how might the individuals' conformity and social supervisory mechanism vary remains a question. Of the entire questionnaire, 7 questions are considered to be critical - 2 on general judgments and 5 on specific scenarios. We attempted to look for any new-established conformity during the outbreak with the help of these questions. In order to observe whether specific group identify exists, the two general questions referred to reference topics with the five scenario-based questions functioned as a supplement. The respondents were asked to rate on a 5-point scale for all seven questions ( 1 =strongly disagree; 5 =strongly agree). Apart from two general questions targeting civic attitudes on mask-wearing, the five scenario-based questions contain various public or private situations. These differentiated scenarios could be further broken down into three categories: driving, trashemptying and smoking. Here, covert controlled measurement on subject behaviors under specific circumstances was then made to investigate whether civic supervision was built based on moral judgments or instead, blind obedience and existing group pressure. In this study, we tried to answer this question using the five specific questions as the main design objectives and the first two general ones as supplementary references.

The questionnaire ends with two open-ended questions, asking for individuals' experiences during COVID-19 and their strategies to cope with anxiety. These could provide additional qualitative data for the study.

\subsection{Data analysis}

Of all 743 responses, ratings on the seven critical questions were analyzed through the mean and distribution of the data. A bar chart and violin plot presented the relationship between individual behaviors and scenarios.

\section{Results}

\subsection{Background characteristics}

758 people agreed to participate, but 15 of them held their rights to withdraw during the study, leaving 743 responses statistically analyzed. Of all respondents $(n=743), 51.0 \%$ were males and $47.2 \%$ were females, with $69.4 \%$ of them above age 30 . For a majority of the respondents, their highest degree (including current enrollment) was either high school (19.5\%), junior school (24.0\%) or undergraduate college (42.5\%). Among all, 17.8\% of the respondents reported a habit of frequent smoking, while $15.2 \%$ of them seldom smoked and $65.1 \%$ had not smoked at all. (Table 1) 
Table 1: Background characteristics of the respondents $(n=743)$

\begin{tabular}{|l|l|}
\hline & $\%$ \\
\hline Gender & $51.00 \%$ \\
\hline Male & $47.24 \%$ \\
\hline Female & $1.75 \%$ \\
\hline Prefer not to answer & \\
\hline & \\
\hline Age & \\
\hline$<25$ & $18.30 \%$ \\
\hline $25-40$ & $33.24 \%$ \\
\hline $41-50$ & $24.50 \%$ \\
\hline$>50$ & $21.53 \%$ \\
\hline Prefer not to answer & $2.42 \%$ \\
\hline & \\
\hline Education level (including currently enrolling) & \\
\hline Junior high school or below & $7.80 \%$ \\
\hline Senior/secondary/vocational school & $19.52 \%$ \\
\hline Undergraduate/college & $66.49 \%$ \\
\hline Graduate or above & $4.58 \%$ \\
\hline Prefer not to answer & \\
\hline & \\
\hline Habit of smoking & $17.77 \%$ \\
\hline Frequently & $65.14 \%$ \\
\hline Occasionally & $1.88 \%$ \\
\hline Never & \\
\hline Prefer not to answer & \\
\hline
\end{tabular}

\subsection{Information relating to COVD-19}

Despite 6 respondents who chose not to answer this question, 8 respondents (out of 737) reported that at least one of their family members or friends tested positive for the coronavirus. Additionally, 81 respondents admitted that they would feel anxious and stressed if they occasionally cough or experience respiratory problems. When being asked to come up with two major behaviors that are largely responsible for the COVID-19 transmission, 638 respondents mentioned mask-wearing, while 572 mentioned keeping distances between people in public. Furthermore, while most of them started to wear masks and avoid social activities after seeing reports in social media and government announcements (594 for social media; 605 for government announcements), participants also stated that these two channels had caused their greatest anxiety (611 for social media; 356 for government announcements).

\subsection{Responses for rating questions}

As presented in Fig.1, $\mathrm{X}$-axis represents various scenarios and $\mathrm{Y}$-axis represents the probability of data distributing on any value. Given that the violin plots for G1 and G2 are wider at both ends and skinners in the middle, people are likely to have clear and definitive adjudication yet limited toleration when dealing with general value judgment questions. Conversely, this is schematically shown in Fig.1 that rating distributions for S1-S5 are visibly different from those for G1-G2, suggesting that civic criticisms for not wearing masks are comparably moderate in some specific real-life situations which are usually considered as within private sphere.

Fig. 2 is a common bar chart showing mean ratings given by all respondents for general and specific scenario-based questions. The mean ratings range from 1 to 5 , with 1 representing strongly disagree and 5 representing strongly agree. With the mean rating for G1 the highest and for G2 the lowest, it demonstrates the tendency that people have clear and unequivocal attitudes towards unspecific judgments. Among S1 to S5, the mean rating for S3, which is associated with not wearing masks in private cars with windows open, is the highest. It is important to note that the mean rating for S1 much higher than that for S2, considering there is no significant change in 
scenarios. As presented by Fig. 2, there is little difference between the mean rating for S4 and S5, even when S4 is considered within the public sphere and S5 within the private sphere. This may raise concerns about the explanations of the supervisory mechanism.

In comparison to Fig. 2, Fig. 3 is a bar chart exhibiting mean ratings given by the smoking population, which are overall higher than that given by all the respondents.

For general questions, there is little difference in mean rating for G1 between Fig. 2 and Fig. 3, whereas that for G2 is more explicit, with the mean rating given by the smoking population 0.17 points. Among the five scenario questions, the mean ratings given for S1 and S3 are especially higher in Fig. 3 than Fig. 2, indicated by a 0.18-point and 0.12-point increase, respectively. Both two questions are considered as car-relating. Furthermore, there have been significant increases in the mean ratings for S4 (0.72 points) and S5 (0.62 points) from Fig. 2 to Fig. 3, which means that the smoking population demonstrated significantly higher tolerance regarding scenarios involving smoking with masks off.

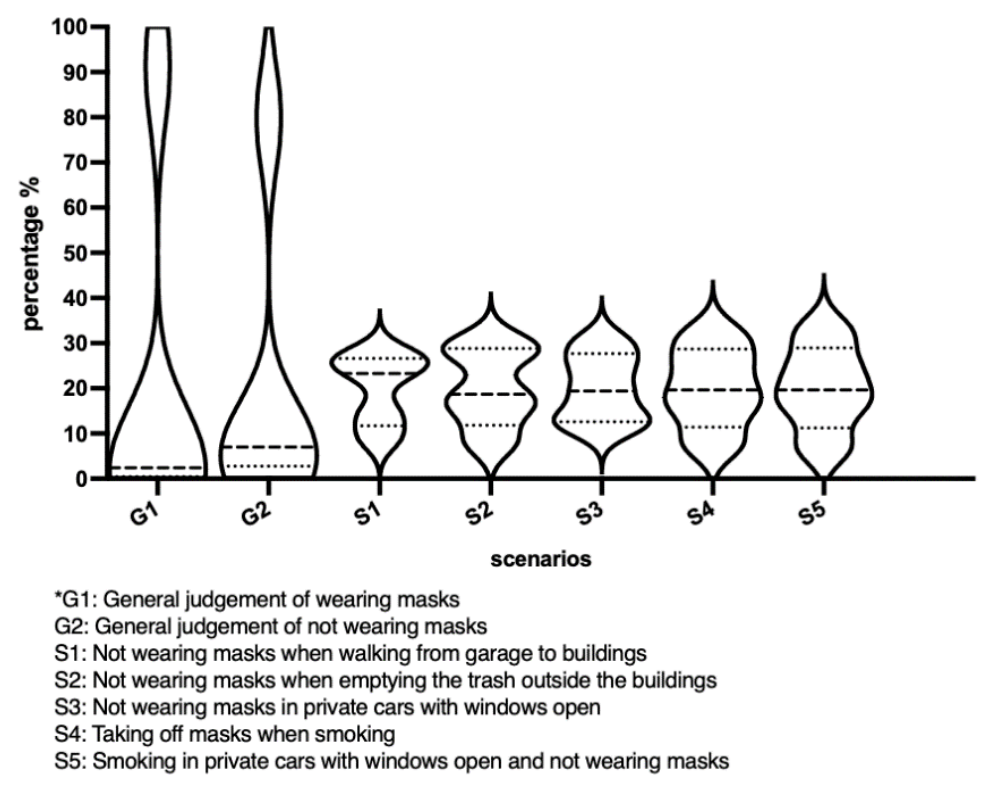

Figure 1. Rating distribution across respondents $(n=743)$.

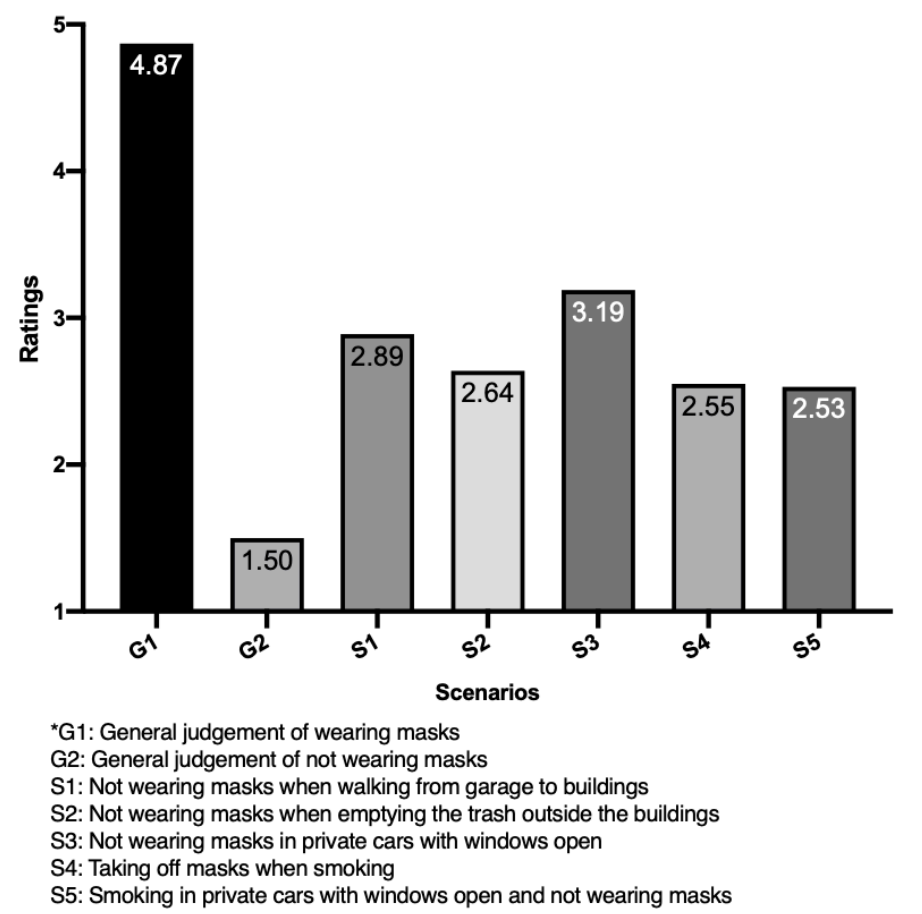

Figure 2. Mean ratings for general and specific questions $(n=743)$. 

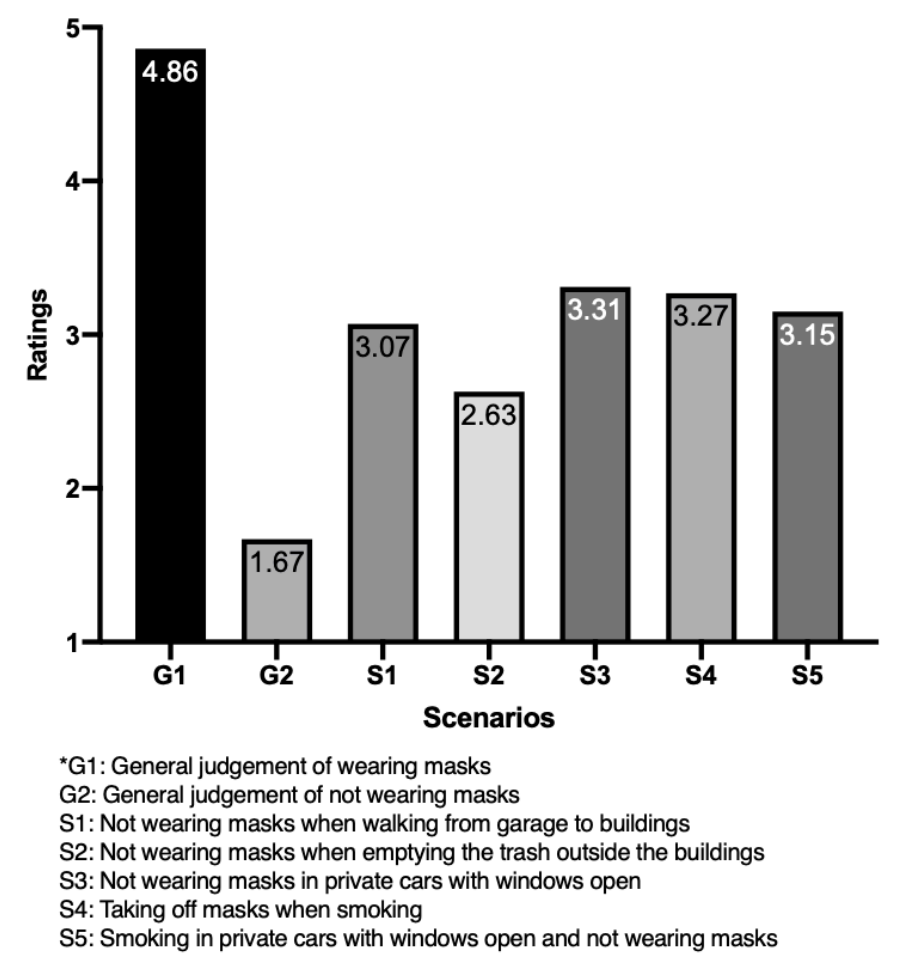

Figure 3. Meaning ratings for general and scenario questions given by the smoking population $(n=245)$

\section{Discussion: public sphere vs. private boundary}

Supposing that differences in social conformity as well as a civil supervisory mechanism with respect to distinct situations exist in the forms of the public or private sphere, then the basic findings could be explained by drawing out the corresponding influences of the public or private sphere.

The questionnaire uses two general questions and five specific ones as a contrast to show that social conformity differs under different contexts related to the public sphere and private ones.

The two general questions, rating for people who agree or refuse to wear masks, overall focus on theoretical and abstract scenes. They mainly describe the public attitude towards the prevention of COVID-19 and strengthening awareness of personal respiratory hygiene. Therefore, among the seven critical questions, these two questions could be viewed as the representatives of the public sphere, which covertly target social supervision power. The five scenario-based questions designed after the general questions, on the other hand, consist of public scenes involving specific real-life actions. According to the data, ratings for the scenario questions did not show a continuation of public-opinion-guided social supervision.

This controversy could be further explained by the private factors involved in the scenario questions. Conventionally, the distance between the garage and the building (S1) is often considered private. More specifically, Leaving the garage then heading to the building could be understood as the transition from either public space to private or the opposite, from private to public. The whole process of S1 involves three stages, which is, someone in his car/garage, someone walking in the open street/public elevator or corridor and then someone at home/his lawn. That is to say, when answering question S1, our testees have their own imaginations on this publicprivate transition and choose the focus among these three stages, which are from a private one to the public then back to private space. The public stage is that one walking in the open street or corridor between two private ones could be easily ignored. By choosing the focus on their own, no matter consciously or not, testees show how their cognitions have affected the intensity of civil supervision on wearing masks. The hidden choices are made by those who grade those questions while S1 question does not take its side.

Similarly, emptying trash outside the buildings (S2) is normally considered to be related to 
private space as well. The whole process of this action includes two stages, which are, being in a building then emptying trash in the open air and the return journey. Therefore, S2 could be deemed as the transition between private space and public. The lower rating(2.64) of S2 compared to $\mathrm{S} 1$ (2.89) shows that it is likely when picturing the situation, the respondents failed to take the private stage into account and merely focus on the public one, that is dumping outside in public. The respondents' cognition unconsciously changed their attitude towards certain circumstances, leading to less tolerance and stricter supervision on mask-wearing. Moreover, ratings on S2 show the only consistency between all of the respondents and smokers, which has a deviation of 0.01(2.63-2.64). This consistency implies that though not all agree on whether it is a public or private behavior when empty trashing outside, the imaginary basis for people to make judgments are stable and unaffected by factors such as smoking.

As the only rating scenario that is higher than 3(3.19), S3 depicts something different. If a judgment goes over the circumstance thoroughly, not wearing masks in private cars with windows open, as suggested in S3, seemingly implies the core notions, both private and public space in the same way. However, the detail through which this scenario is designed makes the very difference that the behavior or the actor remains still and not moving between private and public spaces. Private cars conceptually represent the private boundary since by law, they are part of one's private property. While opening the windows, the scenario also interferes with the public space. For example, due to ventilation, people outside in public might be exposed to potentially dangerous situations if the people inside private cars who do not wear masks are occult patients. That is to say, the sitting-still car driver remains in his private property while his car turns into the vehicle that carries potential infection to public spaces. When answering this question, the testees choose their focus among these two perspectives, and in most cases, people are likely to undervalue the possible negative consequences behind the action of "opening windows"or even totally ignore this public space. The ratings given for the scenarios reflect people's underlying judgments while attempting the questions, which reveal civic habitual thinking patterns tend to be approved when a certain line of private property is drawn.

The last two questions, S4 and S5 involve additional smoking behaviors. The question S4 itself does not specify the exact boundaries; it involves - people could take off their masks and smoke in both public and private space. Nevertheless, the action of "taking off masks" tends to make one think of the public space because at home or in other private places, the likelihood of wearing masks will be significantly lower. Given the mean rating given by all respondents for this question the second-lowest among all five scenarios (2.55), we could cautiously conjecture that when valuing S4, people are more likely to image situations within the public space, for instance, taking off masks and smoking on the street, which will then be associated with "intolerant scenarios". Yet as the mean rating given by the smoking population is significantly higher (3.27), it seems that for S4, the smoking factor has influenced people's common judgments regarding the public and private space. People eventually grade the question based on their own understanding and focus, while the question of S4 itself remains neutral.

The question S5 is mostly similar to S3, with the only difference in the additional smoking behavior. S5 can also be considered to be both private and public — private cars represent the private boundary and opened windows represent interference with the public space. Interestingly, the mean ratings are given by all respondents and the smoking population is lower than those given for S3, with the difference particularly noticeable in Fig.2. Compared with S3, ratings given by all respondents show a deviation of 0.64 (3.19-2.55), while those given by the smokers exhibit a deviation of 0.16 (3.31-3.15). Besides, despite being potentially more private than $\mathrm{S} 4$, both groups spontaneously rated the scenario in S5 as less tolerable. For all of the respondents, the mean rating for S5 is $0.02(2.53-2.55)$ lower than that for S4, and for the smoking population, the difference $(0.12)$ is even larger. Thus, it may suggest that though being slightly different for diverse groups, by adding the smoking scenario, people have shifted their focus away from the public and private space and pay more attention to the consequences of the smoking behavior itself. By narrowing down ones' focus, people rated the question based on personal attitudes towards smoking behavior 
and its impact instead of the whole picture.

Overall, the differences in rating distributions and mean ratings given for S1-S5 may suggest that civic criticisms and supervisions for not wearing masks are comparably moderate and flexible in private boundary situations.

\subsection{Habit and group pressure}

All scenario questions may not be regarded as typically private; instead, they are more likely to be deemed as a mixture of public and private space. Therefore, the apparent differentiation in attitudes could be explained from another viewpoint.

While analyzing data presented in Fig. 2, we speculate that the comparison between S1 and S2, having a deviation of 0.25 (2.89-2.64), is indicative of lack of rational judgment and the possible existence of group pressure. Conventionally, in China, the distance of walking from the garages or parking lots to buildings is longer than that of trash cans from buildings and might take more time. Therefore, from this standpoint, not wearing masks when walking from garages to buildings seems to have a higher risk of spreading the virus due to prolonged contact with pedestrians and the environment. However, the present evidence of contrary ratings introduces a possible contradiction. This may imply that public persistence of wearing masks is derived from social identification of behavioral norms, propagation of mainstream media and social conformity instead of critical thinking.

When the boundary between public and private is obscured in certain scenarios, people tend to make judgments depending on long-standing habits. S4 and S5 in Fig. 2 have the lowest ratings among all special situations. The conjecture can address that the behavior of smoking has broken the boundary between private and public. Hence, the results show that in the context of smoking, mask-wearing no longer seems to be the major determinant that impacts respondents' ratings. It appears that people tend to be generally negative and less tolerant of violations relating to smoking behaviors. Nevertheless, consistent with all scenarios, S4 and S5 only demonstrate situations where people do not wear masks. What's more, unlike other questions, taking off masks while smoking should be more justifiable and is supposed to arouse more empathy since these two behaviors are unlikely to be concurrent. The unexpected tendency shown by data could then be attributed to citizens' inherent reliance on social habits. As the public is generally less tolerant towards the smoking population, due to group pressure, individuals might be inclined to conform to the social habits instead of own rational thinking - giving smoking-related questions low ratings regardless of its underlying cause and intrinsic impact.

\subsection{The smoking population}

Indicated by Fig. 3, the smoking population's mean ratings are mostly higher, especially for S4 and S5. This interesting tendency may reflect more empathy towards scenarios that deal with one's own behaviors. When the scenarios are associated with smoking behaviors, the smoking population is more likely to give higher ratings as they are more capable of sharing their own mental states and understanding others within these situations. Therefore, it could be concluded, though cautiously, that empathy is one of the factors that affect civil supervisory mechanisms in various specific scenarios.

Moreover, according to the questionnaire, it is likely that a special habit has gradually formed among people. This finding may support the differences between Fig. 2 and Fig. 3 since the smoking population might be used to oppose citizens' conventional habit, objecting opinions that smoking behaviors are antipathetic and hence should not be tolerable. As a result, while social conformity of wearing masks is in the process of being established, with two opposite habits, people from different standpoints would hold various attitudes, in this case, giving distinct ratings towards the same scenarios.

\section{Conclusion}

On this basis, the findings of this study suggest that civil supervision would adjust then 
transform according to particular scenarios, which may conclude that Chinese civil supervision concerning the respiratory hygiene issue during COVID-19, though without any strict or distinct supervisory mechanism applied to certain circumstances, has successfully established on its public identification level. This public identification shows high consistency in Zero-tolerance for not wearing masks in public space. However, when it comes to certain circumstances, people tend to balance their attitude according to their understanding of boundaries between public and private. The choices people make to draw the boundaries might be affected by external group pressure and when dealing with various situations relating to mask-wearing, people tend to make judgments depending on the long-established habit. Nonetheless, it is highly possible that making decisions in this way may not cover all potential factors, which sometimes leads to irrational judgments. For most cases, group pressure, habitus and irrational judgments work together, leading to the newly established public conformity astray.

It also needs clarification that by depicting Zero-tolerance for not wearing masks as new conformity of Chinese civil society, this study does not simply assume that single public identification unites all of the dissident groups. Our data indicate that individuals' attitudes towards the same issue could be influenced by empathy. People who are empathetic or have experienced the same situation themselves would be more inclusive even if the matter itself may not be positive. The case is clear when those mask-supervisors happen to be cigarette smokers. Harsh supervision out of public conformity may then soon turns into a flexible improvisation.

\section{References}

[1] Kashif M. Salik, Remittances and COVID-19: Is Pakistan ready for a likely decline in Flows, 2020

[2] Kasperson, R. E., Renn, O., Slovic, P., Brown, H. S., Emel, J., Goble, R., Ratick, S. (1988). The Social Amplification of Risk: A Conceptual Framework. Risk Analysis, 8(2), 177-187.

[3] Cohen, S. (2002). Folk devils and moral panics: The creation of the mods and rockers. Psychology Press.

[4] http://www.nhc.gov.cn/xcs/yqtb/202004/ce78f5575e0d4ef0b4543eb072acebc3.shtml.

[5] Jeremiah, U. WHO raises COVID-19 threat assessment to its highest level -. https://www.vanguardngr.com/2020/03/who-raises-covid-19-threat-assessment-to-its-highest-level/. (2020, March 10).

[6] Antoinette Sayeh and Ralph Chami, The COVID-19 pandemic threatens to dry up a vital source of income for poor and fragile countries, FINANCE \& DEVELOPMENT, VOL. 57, No.2 (2020)

[7] Kashif M. Salik, Remittances and COVID-19: Is Pakistan ready for a likely decline in Flows? (2020)

[8] Wikimedia Foundation. (2020, March 9). Social panic. Wikipedia. https://en.wikipedia.org/wiki/Social_panic.

[10] National Health Commision of the People's Republic of China Reports http://www.gov.cn/zhengce/2020-03/18/content_5492710.htm. 


\section{Appendix I}

\section{Full online questionnaire}

Survey on Public Behaivors During COVID-19

1. Thank you for taking part in this survey! This survey will be collected in the form of anonymity. All information will be kept confidential and only used for academic research. Once the survey is submitted, it can no longer be modified, but you may hold your right to withdraw from this study any time when answering the questions. Thanks for your understanding and support!

- I understand and am willing to take part in this study

- I don't want to take part in this study [TERMINATE]

2. What is your current age?

- Below 18

- 18-25

- $25-30$

- 31-40

- 41-50

- Above 50

- I don't want to answer this question

3. What is your gender?

- Male

- Female

- I don't want to answer this question

4. Do you smoke?

- Often

- Sometimes

- I have never smoked

- I don't want to answer this question

5. What is your highest education level (including currently enrolled)?

- Junior high school or below

- Senior high school/technical secondary school/vocational school

- Junior college

- Undergraduate college

- Master Degree or above

- I don't want to answer this question

6. Did any of your friends or families report testing positive for the coronavirus?

- Yes

- No

- I don’t want to answer this question

7. Recently, have you ever felt worried or anxious for seldomly coughing or discomfort in the repiratory system?

- Yes, I felt worried or anxious for seldomly coughing or discomfort in the repiratory system

- No, I wasn't worried or anxious for seldomly coughing or discomfort in the repiratory system

- No, I did not feel physically discomfortable.

- I don't want to answer this question

8. [M] Which two of behaviors listed below do you think are largely associated with the spread of harmful materials (i.e. fluid, droplets, sources of infection) that may be related to the 
coronavirus?

- Mask-wearing

- Smoking

- Managing pets

- Dealing with domestic waste

- Social-distancing

- I'm not sure about the five behaivors listed above

- Others

9. [M] From which one (or more) of the following channels did you obtain the final information and decided to keep wearing masks and avoid going out?

- Social media/websites

- Government announcements

- Family members/friends

- Community services

- Self-speculation

- Others

10. $[\mathrm{M}]$ Which one (or more) of the following channels brought you the most anxiety?

- Social media/websites

- Government announcements

- Family members/friends

- Community services

- Self-speculation

- Others

11. What is your attitude towards people who insist on wearing masks during the COVID-19? (1=strongly disagree, $5=$ strongly agree)
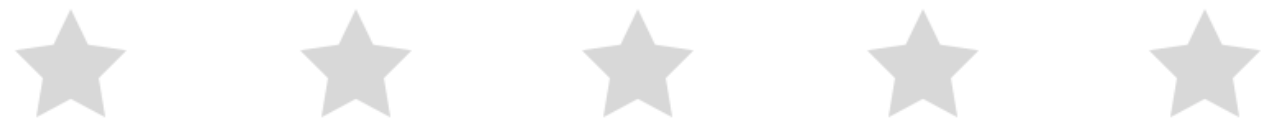

12. What is your attitude towards people who refuse to wear masks during the OCVID-19? (1=strongly disagree, $5=$ strongly agree)
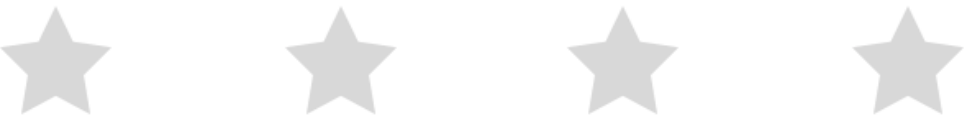

13. One thinks that it is unnecessary to wear masks when walking from the garage to his/her home. What do you feel about this opinion? (1=totally intolerable, 5=totally understandable)
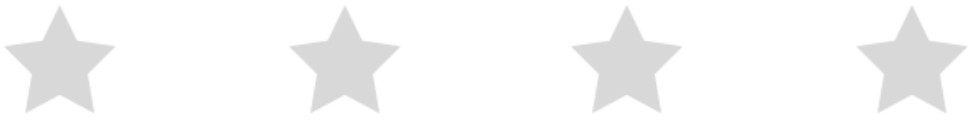

14. What will be your tolerance level if someone does not wear masks when emptying the trash outdoors. (1=totally intolerable, $5=$ totally understandable)
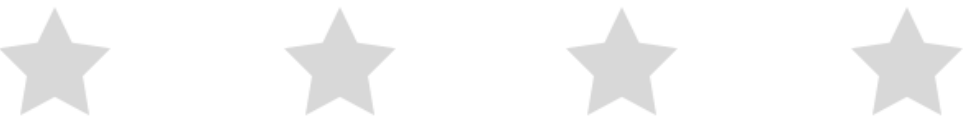

15. What will be your tolerance level if someone does not wear masks in his/her private car with windows open? (1=totally intolerable, $5=$ totally understandable) 

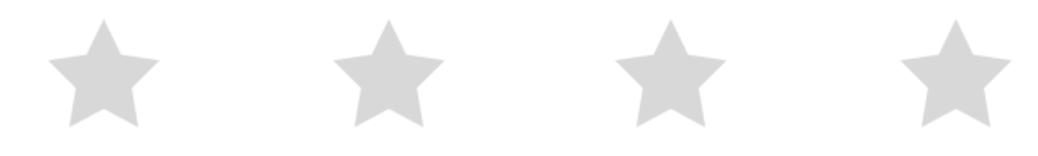

16. What is your opinion or attitude towards the smoking behavior?

- Very supportive

- Supportive

- Tolerable

- Neutral

- Not supportive

- Totally antipathic

17. During the coronavirus, have you ever encountered someone taking off his/her masks for smoking?

- Never

- Sometimes

- Often

- Frequently

- Did not pay attention

18. What will be your tolerance level if someone takes off his/her masks and smokes? (1=totally intolerable, $5=$ totally understandable)
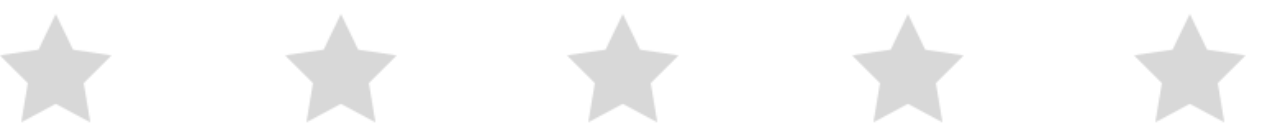

19. What will be your tolerance level if someone does not wear masks in his/her private car and smokes with windows open? (1=totally intolerable, $5=$ totally understandable)
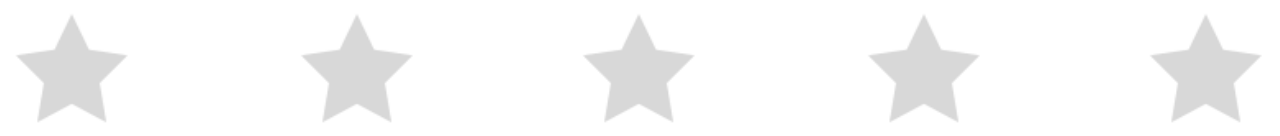

20. [M] If the behaviors or situations listed above acutally happen, which one/ones are you most likely to intervene. (choosing only one option is acceptable)

- Walking on streets without wearing masks

- Do not wear masks when walking from the garage to home

- Do not wear masks when emptying the trash outdoors

- Do not wear maskis in private cars with windows open

- Take off masks and smoking in public

- Taking off masks and smoking in private cars with windows open

- I will not try to interneve any of the situations

21. If the smokers state that smoking will help them to ease anxiety, will you then understand their (smoking) behavior?

- Cannot understand

- Somewhat understandable, but still feel disgusted

- Tolerable/understandable

- I hope that there will be stricter supervisions regarding the smoking issue

- I totally understand because I smoke as well

22. If you do smoke, did this behavior ease your anxiety during the coronavirus?

- Yes, a bit helpful

- Yes, significantly helpful

- Not helpul/no impact

- I do not smoke 
23. [Optional] Have you ever tried to relieve anxiety or pressure during the COVID-19 yourself? In what way?

24. [Optional] Please describe your feelings and experiences during the COVID-19.

CLOSE: Thank you for completing this survey. Stay healthy and have a good day! 